Whether this platform is widely adopted may depend as much on its cost above and beyond standard whole-genome shotgun sequencing as on its technical merit. The authors show that the droplet-barcoded libraries have good genomic coverage and can be used to jointly call and phase SNVs. Despite a modest loss of sensitivity in variant detection, the cost savings are likely to be substantial relative to previous approaches, which require one round of sequencing to discover variants and a second round of sequencing to phase them.

Numerous other sequencing applications could benefit from linked-read data coupled with massively parallel partitioning. Shotgun metagenomics is a prime example, for which similar data generated at a smaller scale have already been used to improve species composition of complex microbial communities and to phase intra-strain variation ${ }^{11}$. Applied to cDNA rather than genomic DNA, linkedread sequencing could be used to deeply assess splice isoform diversity ${ }^{12}$. Finally, similar methods that compartmentalize and prepare
RNA-seq libraries from individual cells ${ }^{13}$ raise the possibility that the approach could be similarly adapted to interrogate gene expression and chromatin state across many thousands of cells in parallel.

\section{COMPETING FINANCIAL INTERESTS}

The author declares no competing financial interests.

1. Zheng, G.X.Y. et al. Nat. Biotechnol. 34, 310-318 (2016).

2. Kitzman, J.0. et al. Sci. Transl. Med. 4, 137 ra76 (2012).

3. Fan, H.C. et al. Nature 487, 320-324 (2012).

4. Jiang, P. et al. Proc. Natl. Acad. Sci. USA 112 E1317-E1325 (2015).

5. Tewhey, R., Bansal, V., Torkamani, A., Topol, E.J. \& Schork, N.J. Nat. Rev. Genet. 12, 215-223 (2011).

6. Browning, S.R. \& Browning, B.L. Nat. Rev. Genet. 12, 703-714 (2011).

7. Snyder, M.W., Adey, A., Kitzman, J.O. \& Shendure, J. Nat. Rev. Genet. 16, 344-358 (2015)

8. Stephens, P.J. et al. Cell 144, 27-40 (2011).

9. Kitzman, J.O. et al. Nat. Biotechnol. 29, 59-63 (2011).

10. Chaisson, M.J.P. et al. Nature 517, 608-611 (2015).

11. Kuleshov, V. et al. Nat. Biotechnol. 34, 64-69 (2016).

12. Sharon, D., Tilgner, H., Grubert, F. \& Snyder, M. Nat. Biotechnol. 31, 1009-1014 (2013).

13. Klein, A.M. et al. Cell 161, 1187-1201 (2015).

\title{
Cas9 loosens its grip on off-target sites
}

\section{Christopher E Nelson \& Charles A Gersbach}

\section{Two new variants of the Cas9 nuclease have minimal activity at off-target DNA sites.}

The excitement surrounding Cas9-an RNAguided nuclease that simplifies editing of nearly any DNA sequence in any organism ${ }^{1}-$ has been tempered by the concern that it can cleave at sites other than the intended target sequence. Such off-target effects may confound experimental results and raise important safety considerations for any potential clinical applications of the approach ${ }^{2}$. Now, two studies, by Zhang and colleagues ${ }^{3}$ in Science and Joung and colleagues ${ }^{4}$ in Nature, report engineered variants of Cas9 with greatly improved specificity. These developments are an important advance toward clinical translation of Cas9-based gene therapies, but also highlight the need for more sensitive methods for monitoring offtarget effects.

Christopher E. Nelson and Charles A. Gersbach are at the Department of Biomedical Engineering, and at the Center for Genomic \& Computational Biology, Duke University, Durham, North Carolina, USA.

e-mail: charles.gersbach@duke.edu
The Cas9 genome editing tool-a complex of the Cas 9 protein and a single guide RNA (sgRNA) - facilitates genome editing because it can be targeted to cleave any DNA sequence of interest by tailoring the sequence of the sgRNA. Cellular DNA repair mechanisms are then exploited to make the desired sequence changes. The first reports of Cas9 off-target activity appeared soon after the initial demonstrations of gene editing, particularly when Cas9 was expressed at high levels. Since then, various strategies for mitigating off-target activity have been proposed ${ }^{5}$. However, they have not been widely adopted owing to various drawbacks, such as requirements for new Cas9 delivery methods, redesigned sgRNAs, multiple sgRNAs per target site, and/or a Cas9 fusion protein in place of conventional Cas9. Because Cas9 is relatively large, fusions to additional domains create challenges for delivery by size-restricted vectors. Moreover, all of these methods still have some residual off-target activity.

The natural function of Cas9 is to recognize and cleave foreign invading DNA as part of a bacterial adaptive immune system ${ }^{1}$. In a previous study, investigators from the Joung laboratory speculated that, to be effective in recognizing foreign DNA, Cas9 likely binds to its targets with more energy than is minimally necessary for activity ${ }^{6}$. This excess binding energy would ensure efficient recognition of weaker target sites and prevent foreign invaders from circumventing detection through mutation. To develop a more specific gene editing tool, they truncated the sgRNA target site to lower its overall binding energy ${ }^{6}$. However, in some cases, truncation also decreased activity at the intended target, and off-target activity, although reduced, could still be observed for some sites. Interestingly, more recent studies suggested that Cas9 with a truncated sgRNA may still bind to some off-target sites without adopting the activated conformation necessary for nuclease function ${ }^{7}$.

Nevertheless, this work established the idea that gene editing specificity could be improved by reducing the strength of the interactions of the Cas9-sgRNA complex with off-target sites to below the threshold necessary for binding while still retaining binding at the target site (Fig. 1a). The present studies ${ }^{3,4}$ build on this concept. Binding of the Cas9-sgRNA complex to DNA relies on both specific and nonspecific interactions. The specific interactions occur between the Cas9 protein and a short DNA sequence known as the protospacer-adjacent motif, and through base pairing of the sgRNA and a DNA sequence of 20 nucleotides. In addition, binding is stabilized by nonspecific interactions between the Cas9 protein and the DNA backbone ${ }^{7}$.

Zhang and colleagues ${ }^{3}$ and Joung and colleagues ${ }^{4}$ both show that by decreasing the strength of these nonspecific interactions through amino acid substitutions, the resulting Cas9 variants are more dependent on correct sgRNA-DNA base pairing, leading to decreases in off-target activity with no significant decreases in on-target activity. Interestingly, the two groups approach this challenge by mutating different regions of Streptococcus pyogenes (Sp) Cas9. Zhang and colleagues $^{3}$ focus on residues that interact with the nontarget DNA strand to generate eSpCas9, whereas Joung and colleagues ${ }^{4}$ alter amino acids that contact the DNA backbone on the target strand to produce SpCas9-HF1 (Fig. 1b). Using unbiased, genome-wide assays, both groups show a reduction of offtarget effects to below detectable levels for several sgRNAs.

The importance of off-target effects is a frequent topic of discussion in the gene editing community, and there is an emerging consensus that the degree to which this issue must be addressed depends on the application. For many uses in basic research, simple controls 
a
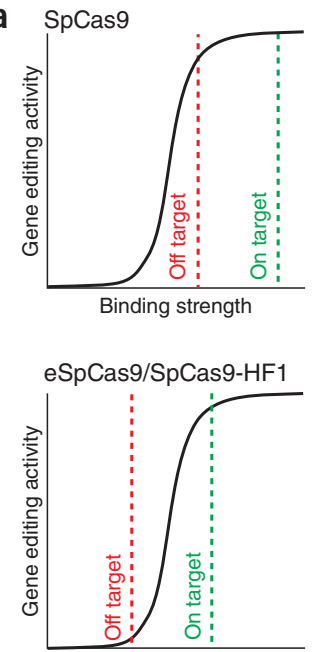

Binding strength

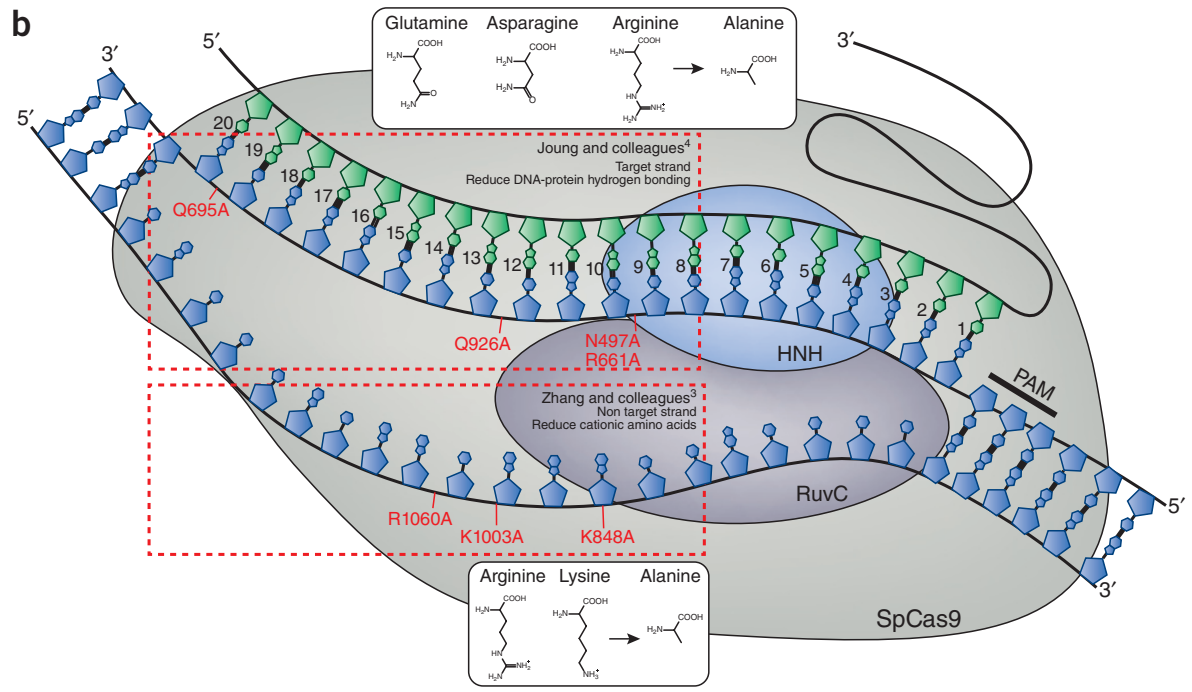

b

Figure 1 Engineering increased specificity into the Cas9 nuclease. (a) Wild-type Cas9 binds both on-target and off-target sites with sufficient energy for efficient gene editing, but decreasing overall binding strength can preferentially reduce activity at off-target sites. (b) Different regions of the Cas9 protein ( $\mathrm{HNH}$ and RuvC) are altered to decrease nonspecific interactions with the DNA backbone. PAM, protospacer-adjacent motif.

may be sufficient ${ }^{5}$. However, additional rigor is warranted for therapeutic applications in which eliminating the potential for off-target modification of tumor suppressor genes or oncogenes is essential ${ }^{2}$. Regardless, the beauty of this work is the simplicity of using eSpCas9 or SpCas9HF1 in place of wild-type SpCas9. There is no barrier to using these variants in any application. Future work will undoubtedly include expanding this approach to Cas9 variants from other species and to epigenome editing tools that use catalytically inactive Cas9 (ref. 8). This work was already begun by Zhang and colleagues ${ }^{3}$ for the Staphylococcus aureus Cas9 (ref. 9), although a complete characterization of these variants remains to be performed.

The new SpCas9 variants are particularly remarkable in that no off-target effects could be found for several sgRNAs using unbiased genome-wide assays. However, an important caveat is the limitation of these methods for detecting low levels of off-target activity $^{5}$. All of these assays depend on nextgeneration sequencing technologies, which have an inherent error rate that varies by target sequence but can be as high as $0.1 \%$ in many cases. Although off-target activity below this level is likely acceptable for many research uses, it could be a concern for therapeutic applications in which hundreds of millions of patient cells are being treated. Similarly, reducing the off-target activity below this level will be particularly valuable for in vivo genome editing in which available delivery methods lead to prolonged Cas 9 expression ${ }^{10}$. Moreover, any differences between eSpCas9 or SpCas9$\mathrm{HF}$ 1, or any further improvements to their specificity, such as combining the amino acid substitutions in each variant, are unmeasurable by current techniques. Thus, increasing the sensitivity of methods for detecting off-target effects is an important goal for the field.

Diminishing off-target effects to below the detection limits of the most sensitive assays available was unimaginable even recently. These new advances promise even further progress toward clinical applications of Cas9 that require the utmost specificity.

\section{COMPETING FINANCIAL INTERESTS}

The authors declare competing financial interests: details are available in the online version of the paper.
1. Doudna, J.A. \& Charpentier, E. Science 346, 1258096 (2014).

2. Maeder, M.L. \& Gersbach, C.A. Mol. Ther. doi:10.1038/ mt.2016.10 (12 January 2016).

3. Slaymaker, I.M. et al. Science 351, 84-88 (2016).

4. Kleinstiver, B.P. et al. Nature 529, 490-495 (2016).

5. Bolukbasi, M.F., Gupta, A. \& Wolfe, S.A. Nat. Methods 13, 41-50 (2015)

6. Fu, Y., Sander, J.D., Reyon, D., Cascio, V.M. \& Joung, J.K. Nat. Biotechnol. 32, 279-284 (2014).

7. Anders, C., Niewoehner, O., Duerst, A. \& Jinek, M. Nature 513, 569-573 (2014).

8. Thakore, P.I., Black, J.B., Hilton, I.B. \& Gersbach, C.A. Nat. Methods 13, 127-137 (2016).

9. Ran, F.A. et al. Nature 520, 186-191 (2015).

10. Nelson, C.E. et al. Science 351, 403-407 (2016).

\section{Research Highlights}

Papers from the literature selected by the Nature Biotechnology editors. (Follow us on Twitter, @NatureBiotech \#nbtHighlight)

Real-time three-dimensional cell segmentation in large-scale microscopy data of developing embryos

Stegmaier, J. et al. Dev. Cell Science 36, 225-240 (2016)

A predictive computational framework for direct reprogramming between human cell types Rackham, O. et al. Nat. Genet. doi:10.1038/ng.3487 (18 January 2016)

Parallel single-cell sequencing links transcriptional and epigenetic heterogeneity Angermueller, C. et al. Nat. Methods doi:10.1038/nmeth.3728 (11 January 2016)

An essential receptor for adeno-associated virus infection

Pillay, S. et al. Nature 530, 108-112 (2016)

Precision tumor recognition by $\mathrm{T}$ cells with combinatorial antigen-sensing circuits Roybal, K.T. et al. Cell doi:10.1036/j.cell.2016.01.011 (28 January 2016)

Engineering customized cell sensing and response behaviors using synthetic Notch receptors Morsut, L. et al. Cell doi:10.1016/j.cell.2016.01.012 (28 January 2016)

Metagenomics uncovers gaps in amplicon-based detection of microbial diversity Eloe-Fadrosh, E.A. et al. Nat. Microbiol. doi:10.1038/nmicrobiol.2015.32 (1 February 2016) 